\title{
Overexpression of whiA in Mycoplasma gallisepticum
}

\author{
E. Tsoy ${ }^{1,2}$, D. Evsyutina ${ }^{1,3 *}$, G. Fisunov ${ }^{1}$ \\ ${ }^{1}$ Federal Research and Clinical Centre of Physical-Chemical Medicine, Moscow, Russia \\ ${ }^{2}$ Pirogov Russian National Research Medical University, Moscow, Russia \\ ${ }^{3}$ Faculty of Bioengineering and Bioinformatics, Lomonosov Moscow State University, Moscow, Russia \\ *e-mail:dar-evsyutina2@yandex.ru
}

Key words: Mollicutes, transcription, WhiA, transcription factor, regulation of gene expression

Motivation and Aim: Mycoplasma species are bacteria that lack cell wall and have reduced genome. There is a scanty knowledge about proteinaceous regulators of transcription in Mycoplasmas [1]. WhiA is a conserved protein in Gram-positive bacteria, and also is present in the Mycoplasmas. This protein has DNA-binding domain. In Streptomyces coelicolor WhiA regulates sporulation [2], but in Bacillus subtilis it influences in chromosome segregation [3] and is involved in cell division [4]. The function of this protein in the Mycoplasmas remains unclear. In this work we searched for targets of WhiA in an avian pathogen Mycoplasma gallisepticum.

Methods and Algorithms: Transposon-based vector pTn4001opt_whiA were constructed for overexpression whiA gene in Mycoplasma gallisepticum S6. Transformation of M. gallisepticum was done by electroporation. All bacteria strains were cultured in liquid medium for exponential phase and cDNA samples were prepared as previously described [5].

Results and Conclusions: The growth rate of trasformants and wild-type bacteria was the same. We selected two independent clones for all experiments. RT-PCR analysis was done for genes involved in all main biological processes and metabolic pathways. The expression of about 80 genes (10\% of all ORFs) was checked. Expression level of nei (mutM), fba and $g l p F$ was changed under whiA overexpression condition. All these proteins can take part in control of redox homeostasis. Fba is a central enzyme of glycolysis but it also has a moonlight function as transcription regulator of catalase and RNA polymerase subunit [6]. Nei (MutM) is a base excision repair enzyme that identifies and eliminates a large variety of oxidized purines from DNA. GlpF is a transporter of glycerol in a pathway for production peroxide. The exact role of WhiA is still not unclear but our results show a further direction of the study.

Acknowledgements: This work was funded by the Russian Science Foundation grant 14-24-00159 "Systems research of minimal cell on a Mycoplasma gallisepticum model".

\section{References}

1. Fisunov G.Y. et al. (2016) Reconstruction of transcription control networks in Mollicutes by highthroughput identification of promoters. Frontiers Microbiology. 7:1977.

2. Kaiser B.K., Stoddard B.L. (2011) DNA recognition and transcriptional regulation by the WhiA sporulation factor. Scientific Reports. 1:156.

3. Bohorquez L.C. et al. (2018) The conserved DNA binding protein WhiA influences chromosome segregation in Bacillus subtilis. Journal Bacteriology. JB.00633-17.

4. Surdova K. et al. (2013) The conserved DNA-binding protein WhiA is involved in cell division in Bacillus subtilis. Journal Bacteriology. JB.00507-13.

5. Mazin P.V. et al. (2014) Transcriptome analysis reveals novel regulatory mechanisms in a genomereduced bacterium. Nucleic Acids Research. 1;42(21):13254-68.

6. Ziveri J. et al. (2017) The metabolic enzyme fructose-1,6-bisphosphate aldolase acts as a transcriptional regulator in pathogenic Francisella. Nature Communications. 8:853. 